\title{
Recent patents on In-Plane Permeability Measurement of LCM Composite Reinforcements
}

\author{
Renaud Gantois, Eric Jourdain and Gilles Dusserre* \\ Université de Toulouse, Mines Albi, CROMeP, Campus Jarlard, 81013 Albi cedex 09, France
}

\begin{abstract}
Optimization of Liquid Composite Molding (LCM) processes using Darcy flow numerical simulation requires inputting accurate reinforcement permeability data. Historically introduced by its author to describe infiltration phenomenon, permeability coming from Darcy's law is usually used in LCM processes as a rheological parameter in order to predict the macroscopic resin motion during the filling stage. Resulting from the flow through a complex fibrous architecture, its measurement is very sensitive to the test conditions due to the high filaments flexibility and mesostructure heterogeneity. Reinforcements are currently anisotropic fibrous media and their in-plane permeability measurement requires specific facilities. Measurements can be performed in transient or steady state conditions, and in one-, two- or three-dimensional configurations. This paper describes the different existing experimental configurations, identification procedures and instrumentation techniques. Advantages and drawbacks of each method are discussed, in the particular case of 2D transient measurements, which are the most representative of Resin Transfer Molding process. Two recent patents using different instrumentation techniques are detailed. They are focused on the simultaneous identification of the in-plane principal permeability values in an anisotropic fibrous reinforcement. Some perspectives are suggested to improve the repeatability of such measurement results.
\end{abstract}

Keywords: Permeability measurement, Fibrous reinforcement, Liquid Composite Molding, Anisotropic porous media.

\section{INTRODUCTION}

The so-called Liquid Composite Molding (LCM) processes, consisting in filling a mold with a resin flowing through a dry reinforcement, are of special interest for the composite industry because of their high automation potential and the reduced storage cost compared to prepreg or laminate technologies. Among them, injection processes, such as Resin Transfer Molding (RTM) and its alternative versions, are widely used in the industry. A recent trend, instigated by vacuum assistance of injection processes, is to develop infusion processes such as Liquid Resin Infusion (LRI) or Resin Film Infusion (RFI). These processes are performed with vacuum assistance and only require a onesided rigid mould, and a vacuum bag. The use of distribution medium speeds up the filling stage and allows producing very large parts at low-cost.

LCM processes optimization using numerical tools requires to model properly the infiltration phenomena through the fibrous medium. This is usually achieved by using Darcy's law, which historically describes the water flow through an isotropic porous medium [1]. Permeability is introduced as the rheological parameter relating flow rate to pressure loss. A three dimensional expression of this equation allows to enlarge its validity domain to anisotropic fibrous media. Nevertheless, the reliability of the LCM filling simulation depends on the accuracy of the permeability data. Permeability being the resultant of the

\footnotetext{
* Address correspondence to this author at the Research Centre on Tools Materials and Processes (CROMeP), Université de Toulouse, Mines Albi, Campus Jarlard, 81013 Albi cedex 09, France;

E-mail: dusserre@enstimac.fr
}

flow around the very complex fibrous architecture, a wide dispersion of the measurement results is foreseeable, increased by the measurement setups complexity.

Whereas injection processes involve essentially in-plane resin flow, infusion processes are characterized by a throughthickness resin flow as a result of the in-plane flow through distribution medium [2]. In the case of thin media such as fibrous reinforcements, the same strategy measurement cannot be applied for in-plane or through-thickness experiment. After a first section about the Darcy's law and the existing permeability measurement techniques, this paper mainly focuses on the patented facilities to measure in-plane permeability in both principal directions.

\section{DARCY'S LAW AND PERMEABILITY PARAMETER}

The phenomenological Darcy's law describes the fluid flow through a porous medium in steady state conditions [1] (historically infiltration of water through a sand bed) by assessing the proportionality between flow rate and pressure loss, assuming fluid incompressibility, perfectly rigid porous medium and that inertia can be neglected (low Reynolds number). A local three-dimensional formulation is useful to replace flow rate by average fluid velocity $\langle\vec{v}\rangle$ by using the pressure gradient $\vec{\nabla} P$ (Eq. (1), where $\mu$ is the fluid viscosity).

$\langle\vec{v}\rangle=-\frac{\overline{\bar{K}}}{\mu} \vec{\nabla} P$

This equation introduces the permeability tensor $\overline{\bar{K}}$ which depends on the architecture of the porous medium (size, number and shape of the pores) and on the different materials (viscosity and surface tension of the fluid and 
wetting angle with the porous medium material). This parameter can be interpreted as the resultant of NavierStokes flow in each possible channel constituted by the pores. In the case of fibrous media, considering the high filaments flexibility and the meso-scale fibrous architecture heterogeneity, the large dependence of permeability value on test conditions leads to widely dispersed measurement results $[3,4]$. This is a very important task to improve LCM processes because permeability influences the process as well as the composite mechanical properties since it determines the material microstructure [5].

\section{SATURATED PERMEABILITY MEASUREMENT TECHNIQUES}

Permeability is historically measured under steady state conditions for hydro-geological application. In particular, petroleum industry has developed many patented facilities to characterize rocks or sand beds [6-8]. Over the years instrumentation advances have allowed the improvement of measurement techniques [9-11]. These methods could be applied to fully saturated non steady state flows [12] which are different as the transient configurations described later. These patents are not detailed here because their application range concerns bulk or granular isotropic materials, whereas only thin samples are in the scope of the present review.

\subsection{Through-thickness permeability}

The most widely used method to measure steady state permeability is the historical one. The fluid is submitted to a one-dimensional pressure loss (respectively flow rate) through a saturated porous medium and the flow rate (respectively pressure loss) is measured. The proportionality coefficient is calculated and the permeability value is deduced. It is performed for chemical or geological applications, and for powder permeability measurements. Its application to thin materials (sheet materials for surgery application sterile packages [13], paper air permeability [14]) is of special interest in the textile industry to measure "waterproofness" and "breathability" of fabrics [15]. This method provides a reliable through-thickness permeability value of fibrous reinforcement and is quite useful for infusion processes simulation. A setup processing such a principle for LCM fibrous reinforcements has been recently patented [16], but it will not be detailed here because it is not in the scope of the paper.

A very interesting approach proposes to continuously measure in-plane and through-thickness permeability of an impregnated fibrous reinforcement against its fiber volume fraction $[17,18]$ while compressing it in the throughthickness direction [19].

\subsection{In-plane permeability}

In-plane steady state permeability measurement is quite different because the sample geometry involves experimental difficulties. The measurement is more sensitive to the fiber deformation due to resin flow because the fabric is only self-clamped in the flow direction. In the onedimensional configuration [20], boundary effects lead to local two- or three-dimensional [21] flows whereas only one-dimensional macroscopic flow is assumed. In such configuration, monitoring assistance is impossible due to the steady state operating conditions. Mould deflection resulting from the large sample area induces deviations on the fiber volume fraction and on the flow section. As a result, theoretical assumptions are no longer valid, making the identification procedure erroneous. In addition, permeability is closely dependent on the fiber volume fraction. Therefore, measurements have to be performed making sure the parameter is well controlled. In two-dimensional configuration $[20,22]$, boundary effects are not so problematic but anisotropic materials could not be tested, excepted if pressure sensors instrumentation is performed [20].

Some authors state permeability deviations according to the measurement technique $[23,24]$. To predict more accurately the filling stage, transient state measurements can be performed, making the test more representative from the real process conditions.

\section{TRANSIENT STATE MEASUREMENT TECHNIQUES \\ PERMEABILITY}

The transient methods consist in monitoring the flow front position to identify the permeability value. They have been introduced in the eighties [25] to provide permeability values to numerical mold filling simulations [26,27]. Both experimental and numerical tools have then been developed to increase reinforcement knowledge and simulation results reliability $[28,29,30]$.

These methods can be performed on dry reinforcements in one-, two- or three- dimensional configuration and provide relevant permeability values for the filling time estimation using numerical tools. Such methods have recently been applied to through-thickness permeability measurement [31], even if most of the studies deal with in-plane permeability. The capillary forces are of great influence in this case [23] and fabric in the vicinity of flow front is not fully saturated [24], what explains the differences observed between transient and steady state measurement results.

\subsection{Permeability identification}

The easiest permeability identification procedure requires constant pressure loss [32,33] or constant flow-rate [20,34] tests. The constant pressure method is the most widely used and will be detailed here.

In 1D configuration, cf. Fig. (1a), mass conservation equation allows to establish that pressure gradient is constant over the sample and Eq. (1) becomes Eq. (2) where $x$ is the flow front position in the Cartesian coordinate system at time $t, \Phi$ is the porosity parameter (ratio between pores and total volume), $K_{x}$ is $x$-direction permeability, $p_{0}$ is the inlet pressure and $p_{f}$ is the flow front pressure.

$\frac{\partial x}{\partial t}=-\frac{K_{x}}{\Phi \mu} \frac{\left(p_{f}-p_{0}\right)}{x}$

If porosity, fluid viscosity and permeability are constant during the test, Eq. (2) can be integrated along time to describe the evolution of flow front position with time, according to Eq. (3). Permeability value is then identified to 

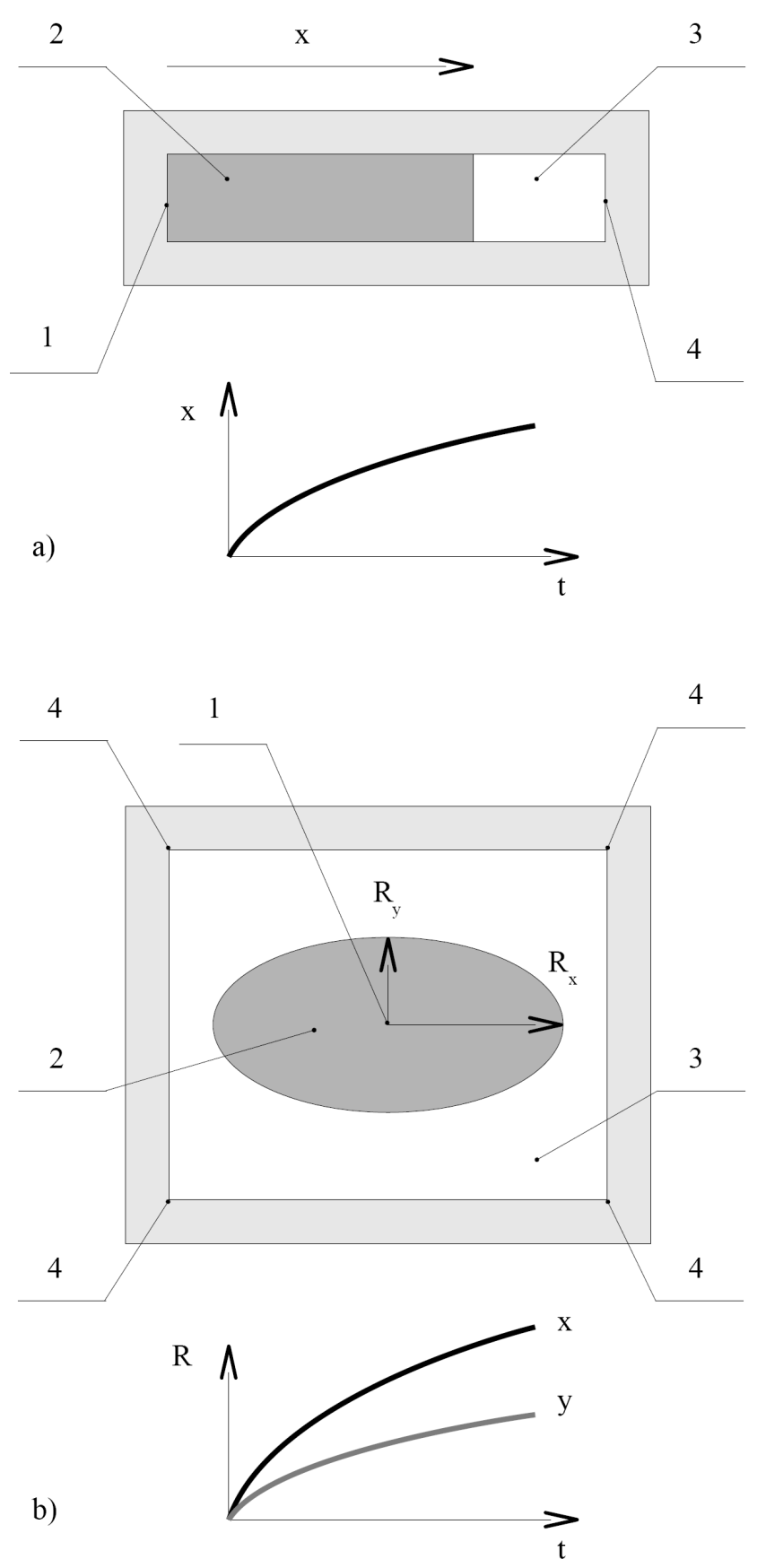

Fig. (1). Schematic view of $1 \mathrm{D}$ (a) and 2D (b) flow front monitoring permeability measurement. 1: injection inlet, 2: saturated reinforcement, 3 : dry reinforcement, 4 : vent.

minimize the differences between experimental and analytical flow front progression.

$x=\sqrt{\frac{2 K_{x}\left(p_{0}-p_{f}\right)}{\Phi \mu} t}$

In 2D configuration, $\mathrm{cf}$. Fig. (1b), an analytical solution exists in the case of isotropic reinforcements. The same reasoning as previous in a cylindrical coordinate system provide the evolution of the flow front radius $R_{f}$ with time depending on the injection inlet radius $R_{0}$ according to Eq. (4) where $K$ is the isotropic in-plane permeability.

$R_{f}^{2}\left[2 \ln \left(\frac{R_{f}}{R_{0}}\right)-1\right]+R_{0}^{2}=\frac{4 K\left(p_{0}-p_{f}\right) t}{\Phi \mu}$

The anisotropic case, which is of great interest for composite reinforcements as UD or woven fabrics, involves elliptical flow front and then requires to resort to an equivalent isotropic system to integrate the Darcy's law [35]. The method will not be detailed further, but both principal permeabilities and orientation of the principal permeability axis can be deduced from the elliptical flow front progression.
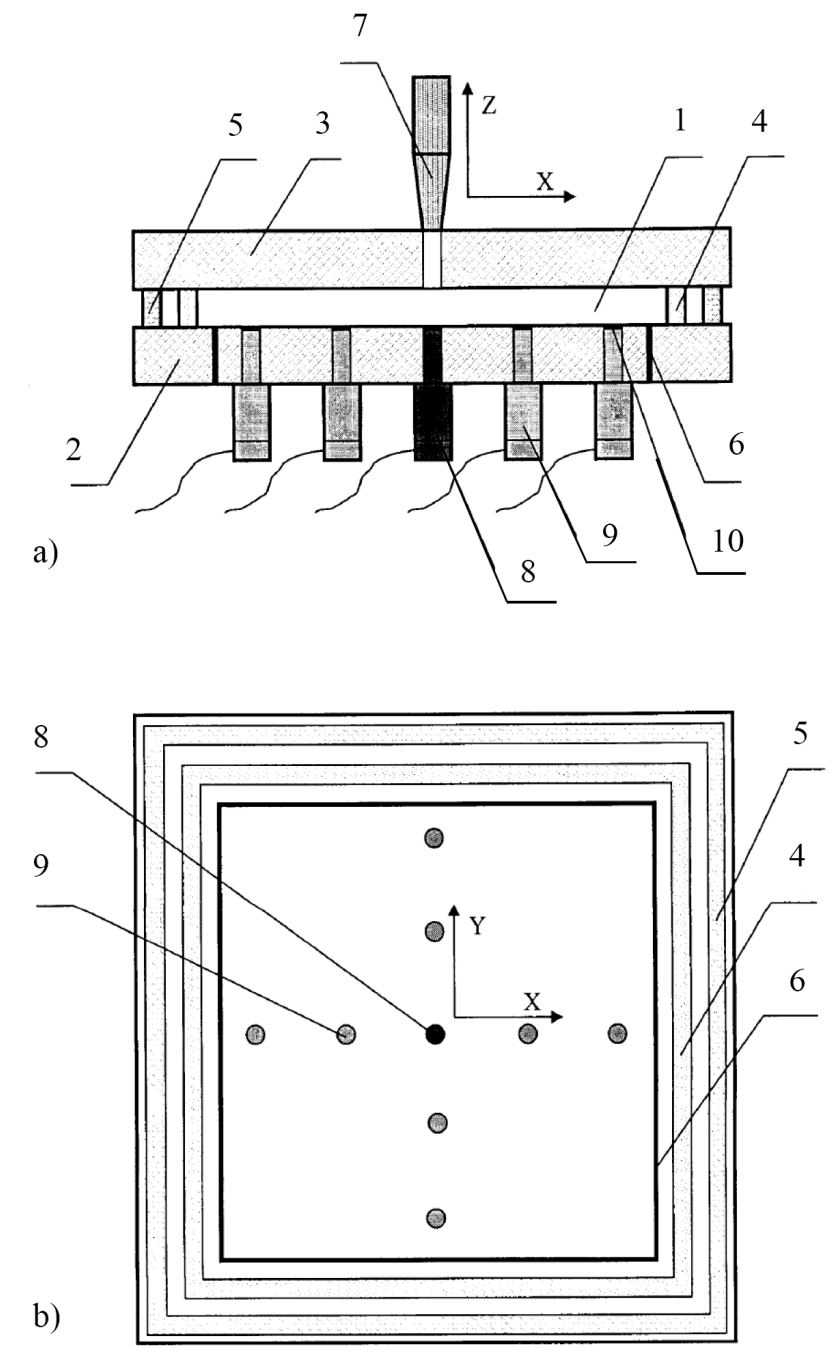

Fig. (2). Patented setup [37] for pressure measurement based permeability identification: a) cross section, b) top view. 1: adjustable gap, 2: lower mould, 3: upper mould, 4: calibrated spacer, 5: inflatable sealing gasket, 6: vent, 7: injection inlet, 8, 9: pressure sensors, 10: measurement cell.

The same reasoning could be extended to 3D configuration in a spherical coordinate system by using the same equivalent isotropic system with a supplementary 
parameter [36]. If identification process complexity is increased, the most critical problem is the sample opacity which requires new instrumentation techniques to monitor the 3D flow front.

\subsection{Flow front monitoring instrumentation}

Optical instrumentation seems to be an evident solution for flow front monitoring and is widely used $[21,32,36]$. It provides very accurate flow front position measurement, including its exact shape at any time. Conventional acquisition period are short enough for composite processing applications and image processing is quite performing to extract the necessary data very quickly. Nevertheless this technique requires transparent moulds (currently made of polymethylmethacrylate, PMMA [32]) and/or vacuum bag [36], and is then very sensitive to mould deflection. The use of thick transparent moulds necessitates the compensation of refraction effect in the flow front monitoring.

For these reasons, other techniques, compatible with thick metallic rigid mould, have been developed using thermistors [33], pressure [34,37] or electrical [38] sensors, or embedded optical fiber [39] with "bare" spots [32] to detect the flow front transit. But even if results can be successfully exploited, the flow front position is monitored discretely and its exact shape remains unknown (in particular, local heterogeneity cannot be investigated). Methods involving pressure measurements provide information about the pressure field in the sample making possible to validate the pressure gradient assumptions [40], but the measurement cell size (about $6 \mathrm{~mm}$ diameter) limits the flow front position measurement accuracy. The measurement cell size of the others techniques is smaller $(<2$ $\mathrm{mm}[32])$ but the flexibility of the sensors make difficult to control exactly their position while infiltrating the sample. An improvement of these instrumentation techniques is possible by the use of capacitive linear sensors [39,41] which allows a continuous accurate monitoring along a few lines placed in beforehand chosen directions.

The last techniques used are inspired by non-destructive testing and are suitable for 3D measurements. Ultrasound transducers $[42,43]$ provide a flow front continuous $3 \mathrm{D}$ measurement, cf. patented setup in [44]. Radioscopy [45] is used as a complement to $2 \mathrm{D}$ optical techniques to monitor a through-thickness projection of the flow front position. Despite the interest of monitoring continually 3D flow front position, the resolution of such techniques is limited and repeatability problems are foreseeable due to the complex experimental setups: encumbrance problems between ultrasound transducers and injection inlet; necessity to use transparent non-metallic (and then few stiff) mould materials, and perfect X-ray source and detector alignment perpendicular to optical instrumentation requirement for radioscopy. Moreover, these methods are limited to thick composite applications. X-ray tomography is quite interesting to monitor the actual flow front 3D geometry, not only a projection as radioscopy, but its processing is not suitable for permeability measurement because of to long acquisition time. Nevertheless it remains a powerful tool to investigate the actual meso-scopic reinforcement architecture which is of great influence on the permeability value [46-48].
A wide instrumentation range is then available for permeability measurements with different advantages and drawbacks. Each one could be selected for a specific application depending for example on pressure measurement necessity, reinforcement compacting level to study fiber volume fraction effects, the sample size and thickness.

\subsection{Infiltration fluid}

The great influence of capillary effects on fibrous media infiltration [49], in particular for high fiber volume fraction applications, has been studied and taken into account to minimize voids formation [50]. Nevertheless it is quite useful to replace resin by another fluid when measuring permeability of LCM reinforcements. Usual resins are very complex fluids and exhibit time and temperature dependent viscosity (due to polymerization reactions) leading to a complex nonNewtonian behavior unsuitable with permeability identification procedures. Moreover most of them cannot be processed at room temperature and cause cleaning as well as occupational health and safety problems. According to $\mathrm{Ma}$ and Shishoo [51] or Luo et al. [52], resin can advantageously be replaced by a similar viscosity fluid (influence of the fluid is not significant compared to the experimental scatter) and Ding et al. [53] link air permeability value to resin permeability value under certain conditions (low Reynolds number) whereas Steenkamer et al. [34] recommend to perform permeability measurements in the exact actual LCM processing conditions. A wide viscosity range is achieved using different perfectly Newtonian oils allowing to simulate different resin types and processing temperatures. Vegetal $[34,36,51]$ or mineral $[33,40]$ oils are often used because their density is very close to those of the polymer resins. Other authors prefer infiltrate the reinforcement with non reactive fluids as dioctylphthalate (DOP) [54] or silicon oil $[55,56]$. Whatever the fluid chosen it is of first importance that its viscosity, density, surface tension and wetting angle are close enough to those of the resin expected to be used in the process wished to be simulated to ensure the reliability of the results. This precaution is necessary to prevent any experimental artifact by taking into account any micro- and meso-scopic flow phenomenon and material interaction in the macroscopic permeability value.

\subsection{Comparison of the different configurations}

Due to the very thin samples under consideration, onedimensional configuration is not suitable for the throughthickness fibrous reinforcement permeability measurement by classical flow front monitoring methods (excepted in [31]). This configuration is sensitive to boundary effects $[57,58,21]$ and to mould deflection, in particular when transparent moulds are required to monitor the flow front position using optical instrumentation. Nevertheless it is very useful to study the behavior of race tracking [21] and to compare transient and steady state permeability values of anisotropic reinforcement because 2D and 3D methods are not suitable in this case for steady state measurements, excepted 2D pressure transducers instrumented methods [20].

Two-dimensional configuration is the most widely used because it is not sensitive to boundary effects [20] and 
provides both in-plane principal permeability values in a same experiment. Nevertheless the larger area of the sample makes measurements still more sensitive to mould deflection. The use of instrumentation techniques, compatible with opaque mould materials and more rigid, is sufficient to evaluate in-plane principal permeability values but information about the actual flow front geometry is lost due to the discretization of the measurement. Experimental data must be interpolated to determine the principal direction of the sample.

Many authors limit their setup to strict 2D flow by cutting a hole in the sample in front of the injection inlet. Flow front position monitoring is then more accurate because it doesn't vary through the thickness direction. But this $2 \mathrm{D}$ method can be enlarged to $3 \mathrm{D}$ measurement by detecting the time the flow front arrives on the mould surface opposite to the injection inlet. The permeability measurement station proposed by Nedanov and Advani [36] involves a transparent mould and a bag to measure $3 \mathrm{D}$ permeability of woven fabrics using a 3D identification procedure. 2D optical flow front monitoring is completed by recording the time when the flow front reaches the bag opposite to the injection inlet. This method is quite performing to monitor the in-plane surface flow front evolution, but it provides only one point in throughthickness direction. As a consequence, the result dispersion is wide and related to the acquisition period, and the validity of Darcy's law cannot be verified. Moreover the use of deformable mould limits the experiment to vacuum assisted processing conditions.

Full three dimensional configuration by ultrasound or radioscopy is quite interesting to measure simultaneously the three principal permeability values. Nevertheless it requires a complex instrumentation with still limited resolution and is limited to thick composites. Nevertheless such techniques must be improved because their use in measurement and model validation or in process monitoring is promising.

\section{PATENTED \\ IN-PLANE \\ PERMEABILITY \\ MEASUREMENT}

If scientist literature provides many descriptions of inplane permeability measurement setups $[59,38,60]$, involving any of the above detailed methods and instrumentation techniques, few are patented. The present section details two recently patented or commercialized facilities involving $2 \mathrm{D}$ flow front monitoring to identify in-plane permeability of LCM reinforcements.

Fig. (2) shows a patented permeability measurement setup using pressure sensors [37]. Pressure measurements are used to detect flow front transit in transient configuration involving non saturated flow but also to obtain local values of the pressure field into the sample. This last characteristic allows to validate the pressure gradient assumptions and to enlarge the application range of the setup to steady state configuration involving fully saturated sample. Measurements can be performed under constant pressure or flow rate conditions. This setup involves rigid moulds and the influence of the sample thickness (using calibrated spacers) and injection pressure can be studied. It is of special interest to study the behavior of the flow under high pressure causing sample deformation, as involved in large parts RTM application. Two principal drawbacks must be underlined. The first one is the discrete flow front monitoring in transient configuration, which necessitates knowing the principal directions before the test to orientate correctly the sample along the pressure sensors axis. The second one is due to the large size of the measurement areas (about $6 \mathrm{~mm}$ diameter). The pressure-time curves are not step function but show a slow increase of the pressure. It is then difficult to estimate accurately the flow front transit time on each sensor.

Fig. (3) shows a facility described in [41] available for commercial application and allowing automated measurements. A high stiffness mould with adjustable gap is instrumented by eight linear capacitive sensors embedded in

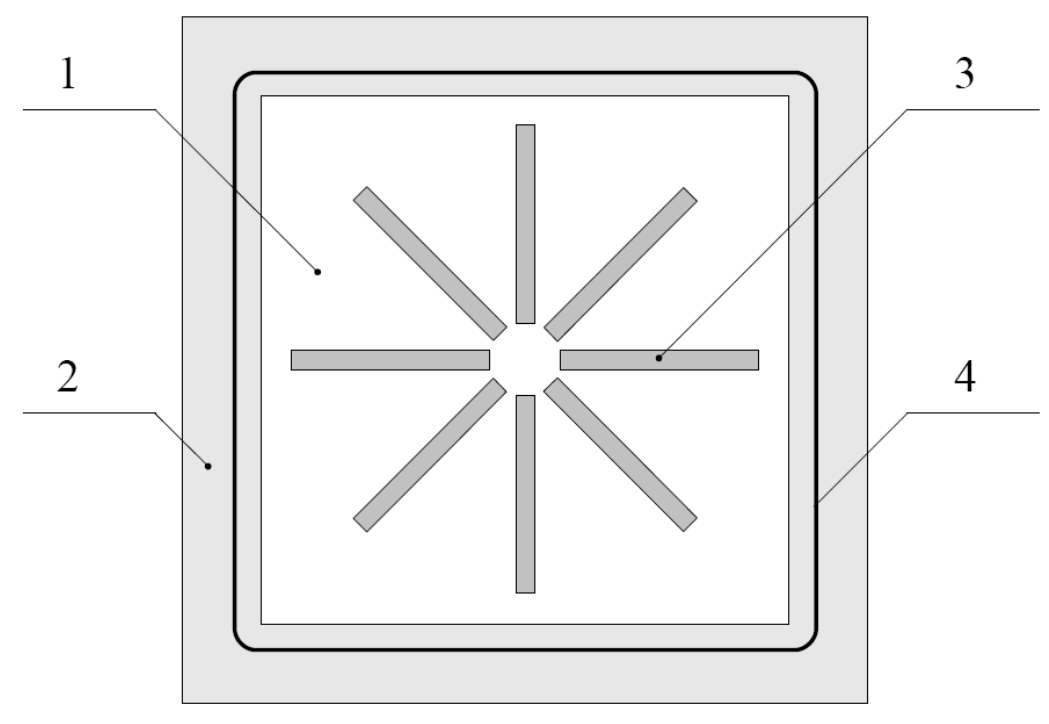

Fig. (3). Schematic view of a commercialized permeability measurement setup [41]. 1: adjustable gap, 2: calibrated spacer, 3: capacitive linear sensors embedded in stellar arrangement, 4: sealing gasket. 
stellar arrangement. The flow front monitoring by this way is continue in a finite number of directions. Interpolating of the flow front position is then possible at any time and fabric principal directions are then determined quite accurately. The station permit to carry out the test in realistic process conditions (high pressures application, heating up to $200^{\circ} \mathrm{C}$, using of industrial resin). It is useful to validate a finite element simulation involving fully coupled thermo-kinetic Darcy flow. This solution seems to be a promising trade-off between stiff mould without anyone local instrumentation and full-field measurement coming with flexible mould. Nevertheless it could be improved by adding pressure measurements to obtain data on the pressure field.

\section{CURRENT \& FUTURE DEVELOPMENTS}

Previous sections have highlighted the wide experimental methodologies range for LCM fibrous reinforcements permeability measurement, involving different flow conditions and almost all the suitable instrumentation techniques, whereas identification procedures are limited to two methods involving pressure flow rate relationship or flow front monitoring. Nevertheless none of the proposed setups seems to be the best candidate for fabric permeability measurement in any conditions.

Some works have been carried out since the nineties to standardize the permeability measurements [61,62], and a glass fabrics database has been developed by the National Institute of Standards and Technology [63]. Nevertheless, no standard has been chosen as the best way to measure permeability of LCM reinforcement. Recently, textile-like solids (rigid specimens with a well known geometry close to a fabric architecture) have been proposed to calibrate permeability measurements $[64,65]$. In order to compare each permeability measurement technique, an important international benchmarking exercise has been started.

Future developments in the field of permeability measurements are foreseeable concerning the instrumentation of the tests. A combination of existing measurement techniques could provide better results, giving the accurate flow front position and making sure the identification procedure is correct. The use of pressure sensors seems to be very interesting not especially to monitor the flow front position but to verify the incompressibility of the fluid while infiltrating the sample. The use of bulk measurement in addition to surface measurement would be interesting to verify that the throughthickness flow in 2D configuration could be neglected, but such instrumentation is often intrusive. Interesting tools for this investigation are long-period or fiber Bragg grating sensor that are very few intrusive in composite applications and have been developed for the monitoring of mold-filling [66] or thermal and mechanical loading in composite parts [67]. Sample properties homogeneity assumptions (thickness, fiber volume fraction and then permeability, but also architecture heterogeneity) have already been investigated [53] but more systematic procedures would be promising to control the tests conditions and then improve their repeatability. The use of precision balance [36] to monitor the fluid quantity injected into the sample would be useful to compare experimental and theoretically evaluated from flow front geometry data. The unsaturated zone size could be estimated and its influence investigated.

Repeatability can be improved by these ways, providing more reliable permeability values. However residual deviations between Darcy flow and realistic industrial flow can be observed, making the measurement a delicate task. This is a consequence of the difficulty to summarize in a single anisotropic homogeneous parameter all the physical phenomena involved when a viscous fluid infiltrates a heterogeneous anisotropic deformable porous media. The deformability of the fibrous medium and its influence on permeability value has been investigated $[68,69]$ as well as the flow in geometrical singularities as mold curvature [7074]. Interesting approach consists in simulating numerically the fluid flow around an idealized fibrous architecture to foresee the permeability value [47,75-79]. This tool is quite powerful to investigate the complex fluid meso-scale flow modification due to deformation without any experimental artifact [80-82]. With the improvement of such tools by including in the simulation statistical heterogeneities and capillary effects, and by modeling properly the actual multiscale fibrous architecture, experimental aspects of permeability evaluation would be limited to fluid characterization and boundary conditions control (as bag deformation in LRI conditions [83]). Nevertheless the methods presented in the paper will still be used but only as validation setup instead of identification.

\section{REFERENCES}

[1] Darcy H. Les fontaines publiques de la ville de Dijon. V. Dalmont, Paris, 1856.

[2] Mathuw R, Advani SG, Heider D, Hoffmann C, Gillespie JW Jr., Fink BK. Flow front measurements and model validation in the vacuum assisted resin transfer molding process. Polym Compos 2001; 22(4): 477-90.

[3] Gauvin R, Kerachni A, Fisa B. Variation of mat surface density and its effect on permeability evaluation for RTM modelling. J Reinf Plast Comp 1994; 13(4): 371-83.

[4] Hoes K, Dinescu D, Sol H, Parnas RS, Lomov S. Study of nesting induced scatter of permeability values in layered reinforcement fabrics. Compos Part A-Appl S 2004; 35(12): 1407-18.

[5] Pearce NRL, Summerscales J, Guild FJ. Improving the resin transfer moulding process for fabric-reinforced composites by modification of the fabric architecture. Compos Part A-Appl S 2000; 31(12): 1433-41.

[6] Boys, G.S.: US2293488 (1938).

[7] Hassler, G.L.: US2345935 (1944).

[8] Hayward, J.T.: US2390252 (1945).

[9] Rose, W.D.: US4506542 (1985).

[10] Shen, J.J.S.: US4773254 (1988).

[11] Bowen, D.G., Davidson Smart, B.G., McLean Somerville, J.: US20046715341 (2004).

[12] Freeman, D.L., Bush, D.C.: US4555934 (1985).

[13] Sorce, P.S., Clifford, E.W.: US4385517 (1983).

[14] Kunitaka, M.: US20026467335 (2002).

[15] Newman, J.R.: US5887477 (1999).

[16] Cauchois, J.P., Philippe, A., Raynal, J., Guissard, S.: FR2900236 (2006).

[17] Comas-Cardona S, Binetruy C, Krawczak P. Unidirectional compression of fibre reinforcements. Part 2: A continuous permeability tensor measurement. Compos Sci Technol 2007; 67(34): 638-45.

[18] Stadtfeld HC, Erninger M, Bickerton S, Advani SG. An experimental method to continuously measure permeability of fiber preforms as a function of fiber volume fraction. J Reinf Plast Comp 2002; 21(10): 879-99.

[19] Comas-Cardona S, Le Grognec P, Binetruy C, Krawczak P. Unidirectional compression of fibre reinforcements. Part 1: A non- 
linear elastic-plastic behaviour. Compos Sci Technol 2007; 67(34): $507-14$.

[20] Han KK, Lee CW, Rice BP. Measurements of the permeability of fibre preforms and applications. Compos Sci Technol 2000;60(1213):2435-41.

[21] Lawrence JM, Barr J, Karmakar R, Advani SG. Characterization of preform permeability in the presence of race tracking. Compos Part A-Appl S 2004; 35(12): 1393-405.

[22] Pomeroy R, Grove S, Summerscales J, Wang Y, Harper A. Measurement of permeability of continuous filament mat glassfibre reinforcements by saturated radial airflow. Compos Part AAppl S 2007; 38(5): 1439-1443.

[23] Lekakou C, Bader MG. Mathematical modeling of macro- and micro-infiltration in Resin Transfer Moulding (RTM). Compos Part A-Appl S 1998; 29(1-2): 29-37.

[24] Bréard J, Henzel Y, Trochu F, Gauvin R. Analysis of dynamic flows through porous media. Part I: Comparison between saturated and unsaturated flows in fibrous reinforcements. Polym Compos 2003; 24(3): 391-408.

[25] Adams KL, Miller B, Rebenfeld L. Forced in-plane flow of an epoxy resin in fibrous networks. Polym Eng Sci 1986; 26(20): 1434-41.

[26] Gauvin R, Chibani M. The modeling of mold filling in resin transfer molding. Int Polym Process 1986; 1(1): 42-6.

[27] Bruschke MV, Advani SG. A finite element/control volume approach to mold filling in anisotropic porous media. Polym Comp 1990; 11(6): 398-405.

[28] Trochu F, Gauvin R, Gao DM, Boudreault JF. RTMFLOT - An Integrated software environment for the computer simulation of the resin transfer molding process. J Reinf Plast Comp 1994; 13(3): 262-70.

[29] Trochu F, Boudreault JF, Gao DM, Gauvin R. Three-dimensional flow simulations for the resin transfer molding process. Mater Manuf Process 1995; 10(1): 21-6.

[30] Gauvin R, Trochu F, Lemenn Y, Diallo L. Permeability measurement and flow simulation through fiber reinforcement. Polym Compos 1996; 17(1): 34-42.

[31] Wu X, Li J, Shenoi RA. A new method to determine fiber transverse permeability. J Compos Mater 2007; 41(6): 747-56.

[32] Ahn SH, Lee WI, Springer GS. Measurement of the threedimensional permeability of fiber preforms using embedded fiber optic sensors. J Compos Mater 1995; 29(6): 714-733.

[33] Weitzenböck JR, Shenoi RA, Wilson PA. Measurement of threedimensional permeability. Compos Part A-Appl S 1998; 29(1-2): 159-169.

[34] Steenkamer DA, McKnight SH, Wilkins DJ, Karbhari VM. Experimental characterization of permeability and fibre wetting for liquid moulding. J Mater Sci 1995; 30(12): 3207-3215.

[35] Adams KL, Russel WB, Rebenfeld L. Radial penetration of viscous liquid into a planar anisotropic porous medium. Int $\mathbf{J}$ Multiphase Flow 1988; 14(2): 203-215.

[36] Nedanov PB, Advani SG. A method to determine 3D permeability of fibrous reinforcements. J Compos Mater 2002; 36(2): 241-254.

*[37] Cauchois, J.P., Philippe, A., Raynal, J., Guissard, S.: FR2900237 (2006).

[38] Liu Q, Parnas RS, Giffard HS. New set-up for in-plane permeability measurement. Compos Part A-Appl S 2007; 38(3): 954-62.

[39] Kueh SRM, Advani SG, Parnas RS. Sensor placement study for online flow monitoring in liquid composite molding. Polym Compos 2000; 21(3): 436-49.

[40] Modi D, Johnson M, Long A, Rudd C. Analysis of pressure profile and flow progression in the vacuum infusion process. Compos Sci Technol 2008, doi:10.1016/j.compscitech.2008.05.026.

[41] Rieber G, Mitschang P. A high-level permeability measurement technique. JEC Composites Magazine 2007; 36: 65-66

[42] Stöven T, Weyrauch F, Mitschang P, Neitzel M. Continuous monitoring of three-dimensional resin flow through a fibre preform. Compos Part A-Appl S 2003; 34(6): 475-480.

[43] Thomas S, Bongiovanni C, Nutt SR. In situ estimation of throughthickness resin flow using ultrasound. Compos Sci Technol 2008; 68(15-16): 3093-3098.

*[44] Stoeven, T.: DE10146323 (2003).
[45] Bréard J, Saouab A, Bouquet G. Permeability measurement of matrix polymer composite reinforcement materials. Eur Phys J AP 1998; 1(2): 269-278.

[46] Badel P, Vidal-Sallé E, Maire E, Boisse P. Simulation and tomography analysis of textile composite reinforcement deformation at the mesoscopic scale. Compos Sci Technol 2008; 68(12): 24332440.

[47] Delerue JF, Lomov SV, Parnas RS, Verpoest I, Wevers M. Pore network modeling of permeability for textile reinforcements. Polym Compos 2003; 24(3): 344-57.

[48] Summerscales J, Russell PM, Lomov S, Verpoest I, Parnas RS. The fractal dimension of X-ray tomographic sections of a woven composite. Adv Compos Lett 2004; 13(2): 115-23.

[49] Dimitrovova Z, Advani SG. Analysis and characterization of relative permeability and capillary pressure for free surface flow of a viscous fluid across an array of aligned cylindrical fibers. J Colloid Interf Sci 2002; 245(2): 325-37.

[50] Ruiz E, Achim V, Soukane S, Trochu F, Bréard J. Optimization of injection flow rate to minimize micro/macro-voids formation in resin transfer molded composites. Compos Sci Technol 2006; 66(34): 475-86.

[51] Ma Y, Shishoo R. Permeability characterization of different architectural fabrics. J Compos Mater 1999;33(8):729-50.

[52] Luo Y, Verpoest I, Hoes K, Vanheule M, Sol H, Cardon A. Permeability measurement of textile reinforcements with several test fluids. Compos Part A-Appl S 2001; 32(10): 1497-504.

[53] Ding L, Shih C, Liang Z, Zhang C, Wang B. In situ measurement and monitoring of whole-field permeability profile of fiber preform for liquid composite molding processes. Compos Part A-Appl S 2003; 34(8): 779-789.

[54] Gourichon B, Deléglise M, Binetruy C, Krawczak P. Dynamic void content prediction during radial injection in liquid composite molding. Compos Part A-Appl S 2008; 39(1): 46-55.

[55] Ouahbi T, Saouab A, Bréard J, Ouagne P, Chatel S. Modelling of hydro-mechanical coupling in infusion processes. Compos Part AAppl S 2007; 38(7): 1646-1654.

[56] Trochu F, Ruiz E, Achim V, Soukane S. Advanced numerical simulation of liquid composite molding for process analysis and optimization. Compos Part A-Appl S 2006; 37(6): 890-902.

[57] Hammami A, Gauvin R, Trochu F. Modeling the edge effect in liquid composites molding. Compos Part A-Appl S 1998; 29(5-6): 603-9.

[58] Hammami A, Gauvin R, Trochu F, Touret O, Ferland P. Analysis of the edge effect on flow patterns in liquid composites molding. App Compos Mater 1998; 5(3): 161-73.

[59] Carter EJ, Fell AW, Griffin PR, Summerscales J. Data validation procedures for the automated determination of the two-dimensional permeability tensor of a fabric reinforcement. Compos Part A-Appl S 1996; 27(4): 255-61.

[60] Morren G, Bossuyt S, Sol H. 2D permeability tensor identification of fibrous reinforcements for RTM using an inverse method. Compos Part A-Appl S 2008; 39(9): 1530-6.

[61] Parnas RS, Howard JG, Luce TL, Advani SG. Permeability characterization. Part 1: A proposed standard reference fabric for permeability. Polym Compos 1995; 16(6); 429-45.

[62] Parnas R. Liquid composite Moulding. Nanser Publishers, Munich, 2000.

[63] Parnas RS, Flynn KM, Dal-Favero ME. A permeability database for composites manufacturing. Polym Compos 1997; 18(5): 623-33.

[64] Morren G, Gu J, Sol H, Verleye B, Lomov S. Stereolithography specimen to calibrate permeability measurements for RTM flow simulations. Adv Compos Lett 2006; 15(4): 119-25.

[65] Morren G, Bottiglieri M, Bossuyt S, Sol H, Lecompte D, Verleye B, Lomov SV. A reference specimen for permeability measurements of fibrous reinforcements for RTM. Compos Part A-Appl S 2009; 40(3): 244-50.

[66] Kueh SRM, Parnas RS, Advani SG. A methodology for using longperiod gratings and mold-filling simulations to minimize the intrusiveness of flow sensors in liquid composite molding. Compos Sci Technol 2002; 62(2): 311-27.

[67] Demirel M., Robert L., Molimard J., Vautrin A., Orteu J.J., Strain and temperature discrimination and measurement using superimposed fiber Bragg grating sensor 13th International conference on experimental mechanics Alexandroupolis, Greece July 2007 
[68] Hammami A, Trochu F, Gauvin R, Wirth S. Directional permeability measurement of deformed reinforcement. J Reinf Plast Comp 1996; 15(6): 552-62.

[69] Tran T, Binetruy C, Comas-Cardona S, Abriak NE. Microporomechanical behavior of perfectly straight unidirectional fiber assembly: theoretical and experimental. Compos Sci Technol 2009; 69(2): 199-206.

[70] Bickerton S, Advani SG, Mohan RV, Shires DR. Experimental analysis and numerical modeling of flow channel effects in resin transfer molding. Polym Compos 2000; 21(1): 134-153.

[71] Bickerton S, Sozer EM, Simacek P, Advani SG. Fabric structure and mold curvature effects on preform permeability and mold fillfing in the RTM process. Part II. Predictions and comparisons with experiments. Compos Part A-Appl S 2000; 31(5): 439-58.

[72] Bickerton S, Sozer EM, Graham PJ, Advani SG. Fabric structure and mold curvature effects on preform permeability and mold filling in the RTM process. Part I. Experiments. Compos Part AAppl S 2000; 31(5) 423-38.

[73] Laval N, Sozer EM, Advani SG. Characterization of permeability around a $90^{\circ}$ corner. Adv Compos Lett 2000; 9(1): 17-24.

[74] Friedman HL, Johnson RA, Gusev V, Neimark AV, Buvel D , Salem DR, Parnas RS. Visualization and quantification of forced in-plane flow through deformed porous media. Polym Compos 1999; 20(5): 613-27.

[75] Loix F, Badel P, Orgéas L, Geindreau C, Boisse P. Woven fabric permeability: From textile deformation to fluid flow mesoscale simulations. Compos Sci Technol 2008; 68(7-8): 1624-1630.
[76] Verleye B, Croce R, Griebel M, Klitz M, Lomov SV, Morren G, Sol H, Verpoest I, Roose D. Permeability of textile reinforcements: Simulation, influence of shear and validation. Compos Sci Technol 2008; 68(13): 2804-2810.

[77] Belov EB, Lomov SV, Verpoest I, Peters T, Roose D, Parnas RS, Hoes K, Sol H. Modelling of permeability of textile reinforcements: lattice Boltzmann method. Compos Sci Technol 2004; 64(7-8): 1069-80.

[78] Nedanov PB, Advani SG. Numerical computation of the fiber preform permeability tensor by the homogenization method. Polym Compos 2002; 23(5): 758-70.

[79] Gokce A, Advani S. Permeability estimation with the method of cells. J Compos Mater 2001; 35(8): 713-28.

[80] Verpoest I, Lomov SV. Virtual textile composites software WiseTex: Integration with micro-mechanical, permeability and structural analysis. Compos Sci Technol 2005; 65(15-16): 2563-74.

[81] Loix F, Orgéas L, Geindreau C, Badel P, Boisse P, Bloch JF. Flow of non-Newtonian liquid polymers through deformed composites reinforcements. Compos Sci Technol 2009; 69(5): 612-19.

[82] Slade J, Sozer EM, Advani SG. Fluid impregnation of deformed preforms. J Reinf Plast Comp 2000; 19(7): 552-68.

[83] Yenilmez B, Senan M, Sozer EM. Variation of part thickness and compaction pressure in vacuum infusion process. Compos Sci Technol 2008, doi:10.1016/j.compscitech.2008.05.009. 